\title{
A novel technique for identification of the segments based on pulmonary artery plane combined with oxygen diffusing discrepancy
}

\author{
Yandong Zhao, Yunpeng Xuan, Jianfang Song, Tong Qiu, Yi Qin, Wenjie Jiao \\ Department of Thoracic Surgery, The Affiliated Hospital of Qingdao University, Qingdao 266003, China \\ Contributions: (I) Conception and design: Y Zhao, W Jiao; (II)Administrative support: J Song; (III) Provision of study materials or patients: W Jiao; \\ (IV) Collection and assembly of data: Y Xuan; (V) Data analysis and interpretation: T Qiu, Y Qin; (VI) Manuscript writing: All authors; (VII) Final \\ approval of manuscript: All authors. \\ Correspondence to: Wenjie Jiao, MD. 16 Jiangsu Road, Qingdao 266003, China. Email: jiaowj@qduhospital.cn.
}

Background: Segmentectomy has been widely accepted as a powerful tool to treat small lung nodules. Despite been commonly used, identify the intersegment plane precisely in visual during the operation still being a complex issue. We described a novel method in minimal invasive thoracic surgery utilizing arterialnavigation combined with oxygen diffusing discrepancy based on the variance of oxygen diffusion in target and reserved segments. We perspectively analyzed 24 cases with small lung nodules of T1N0M0 disease to examine the efficacy of the new method,

Methods: From June 2018 to September 2018, 24 patients with small primary lung cancers underwent segmentectomy at The Affiliated Hospital of Qingdao University. All the patients received minimally invasive procedures and standard anatomical segmentectomy. Ligation of the target segment artery followed by observation and the arterial-navigation line gradually appeared by color discrepancy in the visceral pleural surface. The plane was then testified by inflation-deflation method.

Results: The mean identification time of the intersegments plane was 8 minutes. The residual segments collapsed and stayed pink, meanwhile the target parts remained inflated and turned dark. It allowed a clear identification of the intersegments plane on the surface. All operations were successfully completed. There were no perioperative deaths or major complications. The coincidence rate of arterial-navigation and inflation-deflation technique was 22/24.

Conclusions: The arterial-navigation method is safe and feasible to identify the intersegmental plane in small lung tumor resection.

Keywords: Segmentectomy; segments plane; pulmonary artery plane; oxygen diffusing; minimal invasive surgery

Submitted Aug 21, 2019. Accepted for publication Nov 12, 2019.

doi: $10.21037 /$ jtd.2019.11.42

View this article at: http://dx.doi.org/10.21037/jtd.2019.11.42

\section{Introduction}

Lung cancer is one of the most common causes of cancerrelated death worldwide (1). Lobectomy with systemic lymph node dissection has been considered as a standard surgical treatment for lung cancer (2). In recent years, patients having small peripheral lung nodules or non-small cell lung cancers have been more frequently detected at an early stage. Anatomic pulmonary segmentectomy has been prove as a feasible operative procedure to treat small lung disease with less resection and more lung function sparing $(3,4)$. Furthermore, patients with compromised cardiopulmonary reserve will benefit from this method of approach.

In clinical practice, various methods have been invented to identify the intersegmental plane during pulmonary segmentectomy, but none have been sufficient. The air inflation-deflation technique has been widely used. The 


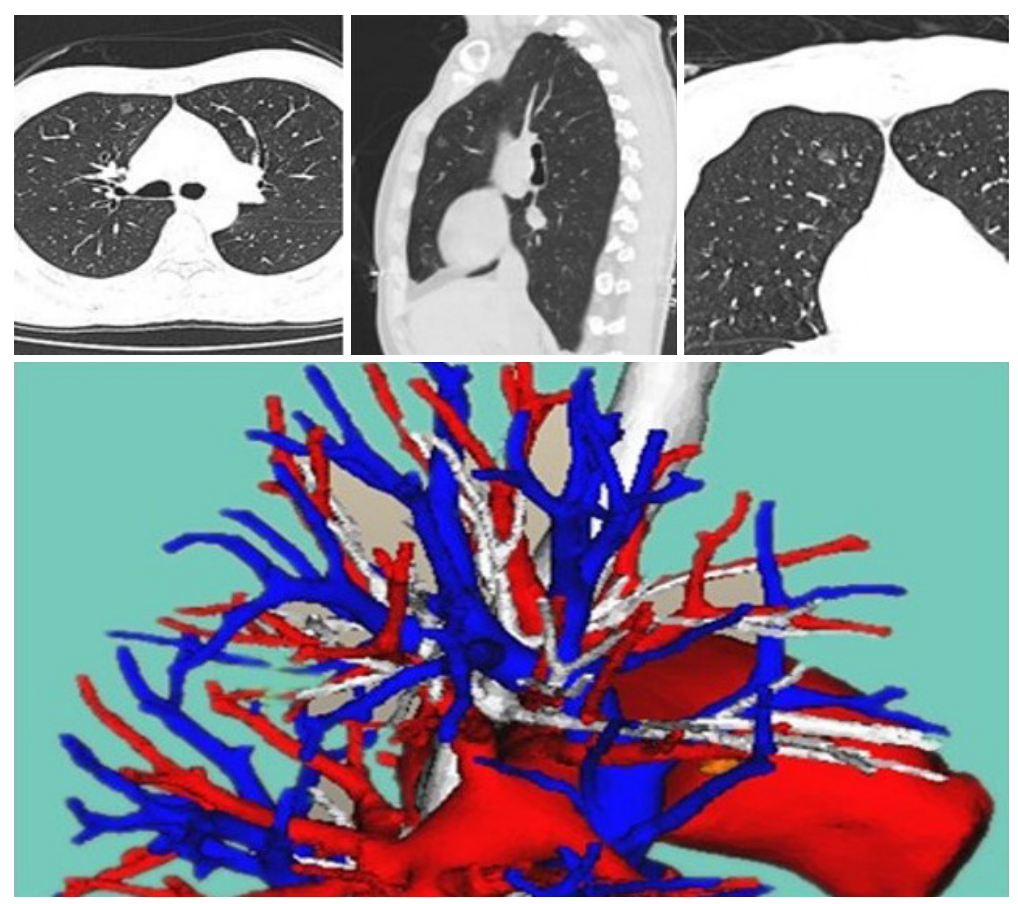

Figure 1 Preoperative 3D computed tomography (CT) and reconstruction.

method was based on the ventilation deference between the target and reserved segments. The obvious disadvantage of this technique is that the expansion of the lung will limit the thoracic cavity and the deflation may be timeconsuming during video-assisted surgery. We have developed a novel arterial-navigation method for intersegmental plane identification. From our previous experience during the segmentectomy procedure, we noticed that the ligation of the target segment artery may cause the imbalance of blood flow in the target and reserved segments. The oxygen diffusion is blocked in the target segment resulting in a semi-complete deflation and ischemia which can be visually identified by a light pink coloring of the parenchyma, while the reserved segments completely collapse and turn dark. The following is a detailed description of our method utilizing the characteristic of arterial-navigated oxygen diffusion.

\section{Methods}

The patients received segmentectomy based on the preoperative clinical findings. No evidence of metastatic disease of the brain, bone, or abdominal organs was discovered. Tumors located in the peripheral lung field with clinical stage I and size $<2 \mathrm{~cm}$ were identified by computed tomography (CT) scan. Informed consent was obtained, and the final decision on resection type would be made during the procedure.

A single-institution, non-randomized study was conducted in 24 consecutive cases of planned anatomical segmentectomy from June 2018 to September 2018. Twenty-four patients underwent anatomical segmentectomy at The Affiliated Hospital of Qingdao University for small primary lung cancer. All the patients underwent minimally invasive surgery (20 video-assisted surgeries and 4 robotassisted surgeries). This study was about the reorganization of mature technologies with no adverse impact on patients. The approval by an ethic committee was not required.

All the patients received a surgical simulation based on preoperative 3D CT work-up (Figure 1). CT images were reconstructed by a team member to obtain the detailed anatomy of the pulmonary vessels to be manipulated during surgery. The target segment artery was defined as the dominant branches accompanying the segment bronchus.

We applied video-assisted or robot-assisted surgical approaches with a minimally invasive method. The surgeon used a mainly video-assisted approach to define the segment boundary and resection of lung parenchyma.

Firstly, the patient was placed in the decubitus position under double-lumen intubation. With the coordination of anesthetist, the lungs were bilaterally ventilated with pure 

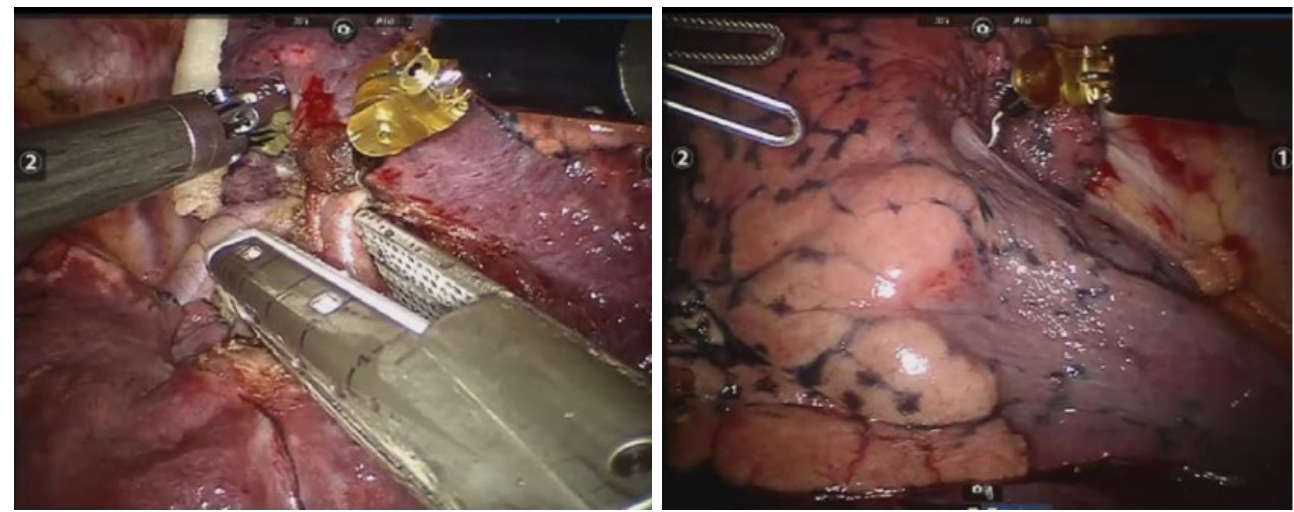

Figure 2 The pulmonary artery for the target segment was exposed and divided through fissure or hilum firstly.

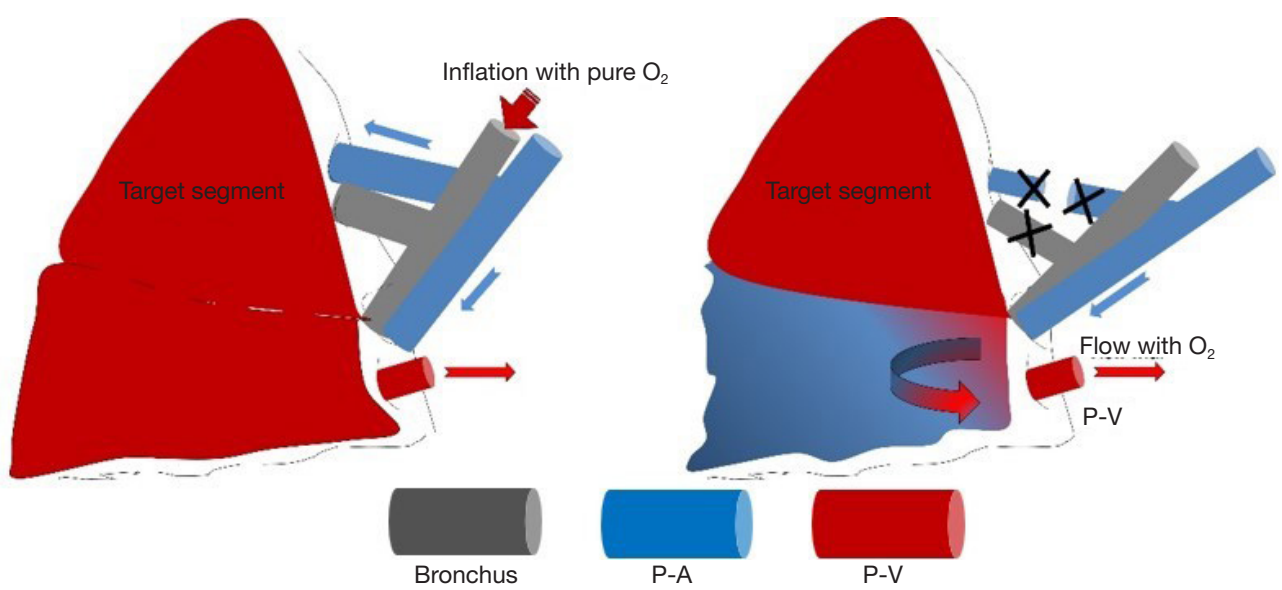

Figure 3 The mechanism of pleural inconsistency for residual and target segments respectively.

oxygen after intubation. Low tide ventilation $(<6 \mathrm{~mL} / \mathrm{kg})$ was conducted immediately after making the first incision (camera port) into the thoracic cavity.

All the other ports were settled (1-2 in video-assisted surgery and 3 in robot-assisted surgery) following the camera port. The pulmonary artery for the target segment was then exposed and divided through a fissure or hilum as the major priority work before the lung totally collapsed (Figure 2). We operated with few parenchyma dissections or indirect palpations in the initial phase. The targeted artery was isolated and ligated with a stapler. Unilateral ventilation was then conducted. Transection of the dominant artery was followed by observation. In minutes, the arterial-navigation line gradually appeared discernible by color and expansion discrepancy, being dark in the reserved area and light in the target area. The inconsistency of collapsed and expanded pleura was also visible (Figure 3). The line on pleura was then marked by electrocautery with a discontinuous point.

The target segment bronchus and vein were then managed in our routine procedure. An air inflation-deflation technique was utilized to verify the arterial-navigation line after the bronchus was cut off. If the two methods presented an identical plane, the resection of parenchyma along the intersegmental pulmonary vein and the marked line of the arterial-navigation plane was completed with staplers.

\section{Results}

Twenty-four patients (12 male and 12 female) with small lung disease underwent anatomic segmentectomy with minimal-invasive surgery at The Affiliated Hospital of Qingdao University without thoracotomy. Patient characteristics are listed in Table 1. All the patients had a diagnosis of malignancy. Anatomic segmentectomy was 
Table 1 Pre-operative characteristics

\begin{tabular}{lc}
\hline Variables & $\mathrm{n}=24$ \\
\hline Age (years) & $64 \pm 13$ \\
Sex, $\mathrm{n}(\%)$ & $12[50]$ \\
Male & $12[50]$ \\
Female & \\
Size of tumor (mm) & $4-15$ \\
Range & $11 \pm 5$ \\
Mean & $1.9 \pm 3.4$ \\
FDG-PET (SUV) & $12 \pm 10$ \\
C/T ratio (\%) & $1,750 \pm 936$ \\
FEV1 (mL) & $2.8 \pm 1.4$ \\
CEA (ng/mL) & \\
Clinical stage, $\mathrm{n}(\%)$ & $22(91.67)$ \\
IA & $2(8.33)$ \\
IB
\end{tabular}

$\mathrm{C} / \mathrm{T}$ ratio, consolidation/tumor ratio; FDG-PET, 2-deoxy-2[18F] fluorodeoxyglucose positron emission tomography; SUV, standardized uptake value.

Table 2 Distribution of the 24 segments

\begin{tabular}{ll}
\hline Cases & Number \\
\hline Right $(n=16)$ & 2 \\
$\mathrm{~S} 1$ & 4 \\
$\mathrm{~S} 2$ & 1 \\
$\mathrm{~S} 3$ & 1 \\
$\mathrm{~S} 1+2$ & 4 \\
$\mathrm{~S} 6$ & 1 \\
$\mathrm{~S} 8$ & 2 \\
$\mathrm{~S} 9+10$ & 1 \\
$\mathrm{~S} 2 \mathrm{~b}+3 \mathrm{a}$ & \\
Left $(\mathrm{n}=8)$ & \\
$\mathrm{S} 1+2$ & 1 \\
$\mathrm{~S} 6$ & 3 \\
$\mathrm{~S} 1+2+3$ & 2 \\
$\mathrm{~S} 4+5$ & 1 \\
$\mathrm{~S} 8+9+10$ & 1 \\
\hline
\end{tabular}

performed on these patients, with 20 undergoing VATS and 4 undergoing robot-assisted surgery. Among the 24 patients, different types of segmentectomies were included (16 right side and 8 left). There were four S2 and S6 segments and one $\mathrm{S} 3, \mathrm{~S} 8$, and $\mathrm{S} 2 \mathrm{~b}+3 \mathrm{a}$ on the right side. Meanwhile, there were three S6, two S1+2+3 (inherent segments), and one left lingual and S8-10 segment on the left side (Table 2). Free surgical margins were pathologically proven, and systemic lymph node sampling was performed in all patients.

The mean identification time of the plane was 8 minutes. The coincidence rate of artery-gas navigation and inflationdeflation technique was 22/24. Segment plane identification in two cases was not very clear due to delayed ligation of the target artery and a total collapse of the lobe.

\section{Discussion}

The candidates for segmentectomy were considered as early stage lung cancer (5). Some retrospective studies have shown segmentectomy has a comparable short and long-term prognosis with lobectomy in selected patients while preserving more lung function (6-8). However, anatomical segmentectomy essentially involves the difficulty of identifying the intersegmental plane inside the lung parenchyma between the targeted and reserved segments and making a precise resection of the target. There is no obvious boundary between segments. The procedure demands that the surgeon have a thorough three-dimensional view of the pulmonary bronchovascular structures.

A number of methods have been reported for identifying the intersegmental plane (9-12). Bronchus navigation methods have been widely used. The deflation method involves clamping the bronchus of the reserved segment and creating a demarcation line with the target. The inflation-deflation involves inflating the whole lobe after clamping or ligating the target bronchus and then deflating the reserved segments. Okada (11) reported a method of selective targeting bronchus high-frequency ventilation under bronchoscope. Changing the ventilation strategy is the essence of the bronchoscopic-navigation method. The plane can be drawn on the pleural surface. However, the ventilation should be conducted by an anesthetist in close cooperation with the surgeon. The selective ventilation requires intraoperative bronchoscopy and adequate time to distinguish the boundary. The technique of using 
Indocyanine green injection to the distal target bronchus is another bronchoscopic-navigation method (9). The target pulmonary segments are immediately stained green, and a clear boundary is visible after the injection. However, the complexity and open airway in the operating field restrict the adoption of this technique.

Vessel-navigated methods have also been tested. The photodynamic diagnosis (PDD) technique (13) uses the key instruments of the D-Light system, which is an excitation light source combined with a camera as the fluorescence sensing endoscope. In this method, the dominant pulmonary arteries are ligated. The patients have indocyanine green injected into a peripheral vein for observation of the lungs under infrared light. This technique can identify the intersegment plane without lung inflation; however, the indesirable use of injection and complexity will impede the application.

The important feature of our method is that it is convenient to apply. There is no additional cost and little support required from other staff. Only the 3D CT image reconstruction and differentiation of the pulmonary artery are depended on during the procedure. It allows a clear identification of the plane owing to the oxygen diffusion rate in the adjacent segments. For a blood flow change, the oxygen exchange in reserved segments will cause lung deflation; meanwhile this process is interrupted in the target segments and lead to inflation. However, the method still has some disadvantages. The boundary can only be distinguished on the pleural surface instead of the parenchyma, and detection is available for a limited period. Furthermore, during VATS or other minimal invasive surgery, the oxygen diffusion discrepancy is not easily visualized in patients with impaired lung function or chronic bronchitis with a deposition of dust.

\section{Conclusions}

Our study suggests that the arterial-navigation method is a simple and easy way to identify the intersegmental plane. The use of this technique allows for the safe and feasible resection of small lung tumors.

\section{Acknowledgments}

None.

\section{Footnote}

Conflicts of Interest: The authors have no conflicts of interest to declare.

Ethical Statement: The authors are accountable for all aspects of the work in ensuring that questions related to the accuracy or integrity of any part of the work are appropriately investigated and resolved. The approval by an ethic committee was not required.

\section{References}

1. Siegel RL, Miller KD, Jemal A. Cancer statistics, 2018. CA Cancer J Clin 2018;68:7-30.

2. Ginsberg RJ, Rubinstein LV. Randomized trial of lobectomy versus limited resection for T1 N0 non-small cell lung cancer. Lung Cancer Study Group. Ann Thorac Surg 1995;60:615-22; discussion 622-3.

3. Nakamoto K, Omori K, Nezu K, et al. Superselective segmentectomy for deep and small pulmonary nodules under the guidance of three-dimensional reconstructed computed tomographic angiography. Ann Thorac Surg 2010;89:877-83.

4. Yu W, Wang Z, Qian L, et al. HRCT features distinguishing minimally invasive adenocarcinomas from invasive adenocarcinomas appearing as mixed ground-glass nodules. Transl Cancer Res 2018;7:1298-305.

5. Inoue M. Careful Indications for Intentional Segmentectomy in cT1aN0 Non-Small Cell Lung Cancer. Ann Thorac Surg 2017;104:1095-6.

6. Song CY, Sakai T, Kimura D, et al. Comparison of perioperative and oncological outcomes between videoassisted segmentectomy and lobectomy for patients with clinical stage IA non-small cell lung cancer: a propensity score matching study. J Thorac Dis 2018;10:4891-901.

7. Nonaka M, Kadokura M, Yamamoto S, et al. Tumor dimension and prognosis in surgically treated lung cancer: for intentional limited resection. Am J Clin Oncol 2003;26:499-503.

8. Watanabe T, Okada A, Imakiire T, et al. Intentional limited resection for small peripheral lung cancer based on intraoperative pathologic exploration. Jpn J Thorac Cardiovasc Surg 2005;53:29-35.

9. Oh S, Suzuki K, Miyasaka Y, et al. New technique for lung 
segmentectomy using indocyanine green injection. Ann Thorac Surg 2013;95:2188-90.

10. Sekine $\mathrm{Y}$, Ko E, Oishi H, et al. A simple and effective technique for identification of intersegmental planes by infrared thoracoscopy after transbronchial injection of indocyanine green. J Thorac Cardiovasc Surg 2012;143:1330-5.

11. Okada M, Mimura T, Ikegaki J, et al. A novel video-assisted anatomic segmentectomy technique: selective segmental inflation via bronchofiberoptic jet followed by cautery cutting. J Thorac Cardiovasc Surg 2007;133:753-8.

Cite this article as: Zhao Y, Xuan Y, Song J, Qiu T, Qin Y, Jiao W. A novel technique for identification of the segments based on pulmonary artery plane combined with oxygen diffusing discrepancy. J Thorac Dis 2019;11(12):5427-5432. doi: 10.21037/jtd.2019.11.42
12. Kasai Y, Tarumi S, Chang SS, et al. Clinical trial of new methods for identifying lung intersegmental borders using infrared thoracoscopy with indocyanine green: comparative analysis of 2- and 1-wavelength methods. Eur J Cardiothorac Surg 2013;44:1103-7.

13. Waseda R, Oda M, Matsumoto I, et al. A novel fluorescence technique for identification of the pulmonary segments by using the photodynamic diagnosis endoscope system: an experimental study in ex vivo porcine lung. J Thorac Cardiovasc Surg 2013;146:222-7. 